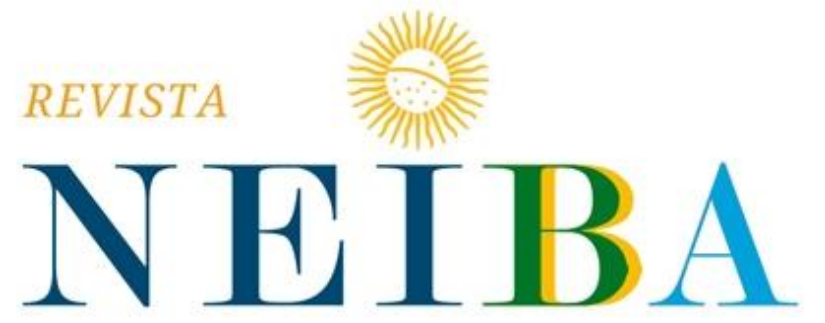

CADERNOS ARGENTINA-BRASIL
Volume 9,2020 , p. $01-23$

DOI: $10.12957 /$ neiba.2020.50600|e50600 I ISSN: 2317-3459

\title{
LOS LÍMITES DEL PROGRESISMO: LA POLÍTICA EXTERIOR DE ARGENTINA Y MÉXICO HACIA AMÉRICA LATINA ${ }^{1}$
}

OS LIMITES DO PROGRESSISMO: A POLÍTICA EXTERNA DA ARGENTINA E DO MÉXICO EM RELAÇÃO À AMÉRICA LATINA

\section{Matheus de Oliveira Pereira ${ }^{2}$}

\section{Marcela Franzoni ${ }^{3}$}

${ }^{2}$ Programa de Pós-Graduação em Relações Internacionais San Tiago Dantas (UNESPUNICAMP-PUC-SP), São Paulo, SP, Brasil. E-mail: matheus.mop@gmail.com ORCID: https://orcid.org/0000-0003-1525-4831.

${ }^{3}$ Programa de Pós-Graduação em Relações Internacionais San Tiago Dantas (UNESP, UNICAMP, PUC-SP), São Paulo, SP, Brasil. E-mail: marcelafranzoni32@hotmail.com. ORCID: https://orcid.org/0000-0002-7280-3318.

Recebido em: 01/01/2020 | Aceito em: 27/04/2020.

\footnotetext{
${ }^{1}$ El presente trabajo fue realizado com apoyo de la Coordenação de Aperfeiçoamento de Pessoal de Nível Superior Brasil (CAPES) - Código de Financiamento 001 y de Fundação de Amparo à Pesquisa do Estado de São Paulo (FAPESP), proceso 2019/05303-8.
} 


\section{RESUMEN}

La elección de Alberto Fernández generó expectativas de una reanudación de las agendas de política exterior que caracterizan el período de la "marea rosa" en América Latina. Desde este punto de vista, Argentina y México actuarían como el eje de un bloque progresista que organiza la resistencia a las agendas de la derecha y propone una reanudación de los mecanismos de cooperación creados en la primera década del siglo XXI. El artículo argumenta que la posibilidad de una política exterior progresista, aunque existente, debe ponerse en perspectiva debido a los desafíos que enfrentan ambos gobiernos. En particular, la situación económica en Argentina y México puede actuar como un obstáculo para iniciativas más vigorosas en política exterior para América Latina.

Palabras-clave: América Latina; Argentina; México.

\section{RESUMO}

A eleição de Alberto Fernández gerou expectativas de uma retomada das agendas de política externa que caracterizam o período da "maré rosa" na América Latina. Desse ponto de vista, Argentina e México atuariam como o eixo de um bloco progressista que organiza a resistência às agendas de direita e propõe a retomada dos mecanismos de cooperação criados na primeira década do século XXI. O artigo argumenta que a possibilidade de uma política externa progressiva, embora exista, deve ser posta em perspectiva devido aos desafios enfrentados pelos dois governos. Em particular, a situação econômica na Argentina e no México pode ser um obstáculo para iniciativas mais vigorosas em política externa para a América Latina.

Palavras-Chave: América Latina; Argentina; México. 


\section{INTRODUCCIÓN}

En noviembre de 2019, poco más de una semana después de vencer las elecciones, Alberto Fernández eligió a México como su primer destino internacional como presidente electo de la Argentina. En un primer momento, se presentó el viaje bajo la consigna de profundizar las relaciones bilaterales con un importante aliado, sobre todo en los ámbitos de comercio y medio ambiente. En ese sentido, Fernández tuvo una agenda llena de encuentros con líderes políticos y empresariales, buscando la ampliación del mercado de los productos argentinos en México y atracción de inversiones (Observatorio de Política Exterior Argentina, 2019b).

Además de la promoción comercial, la visita a México se convirtió en un momento de presentación más detallada de las intenciones del nuevo presidente argentino respecto a la política exterior. Después de una reunión privada con el presidente Andrés Manuel López Obrador, Fernández reiteró junto a la prensa su voluntad de darle a la política exterior argentina una impronta que él definió como "progresista" (Dinatale, 2019). En este sentido, la elección de México como primer destino internacional no fue casual, ni tampoco puede ser limitada a la dimensión económica.

En un contexto regional marcado por una amplia prevalencia de gobiernos de derecha y extrema-derecha, México se destaca justamente por ser gobernado, desde 2018, por López Obrador, un político de larga trayectoria en la centroizquierda. Lo que propone Alberto Fernández en términos de política exterior es contraria de la tendencia regional actual, caracterizada por la rápida erosión de las iniciativas gestadas por sus antecesores en el período de la llamada "marea rosa" (Panizza, 2006). Por esta razón, las relaciones con México adquieren una dimensión relevante, como un punto de apoyo intuitivo para la búsqueda de estos intereses.

La posterior derrota del Frente Amplio en Uruguay y la caída de Evo Morales en Bolivia, refuerzan la posición de México y Argentina como los artífices posibles de una oposición a la agenda desarrollada por los gobiernos de derecha en la región. Tan pronto Fernández venció las elecciones, el presidente venezolano Nicolás Maduro afirmó que Fernández y Obrador estaban "Ilamados" a encabezar un frente progresista y antineoliberal (Observatorio de Política Exterior Argentina, 2019a). Lejos de ser una 
posición aislada, la afirmación de Maduro repercutía en las respuestas dadas por los principales líderes de izquierda en la región, que saludaron a Alberto Fernández asociando el resultado electoral de Argentina a una victoria de la "patria grande" (Observatorio de Política Exterior Argentina, 2019a).

Fernández y Obrador contestaron de modo más ponderado a este "Ilamado", haciendo explícito que no pretendían ser definidos como oposición de nadie, pero reafirmaron el interés común de fortalecer la coordinación política en América Latina. En un texto publicado en los medios de comunicación, el subsecretario para América Latina y el Caribe de la Secretaría de Relaciones Exteriores de México, Maximiliano Zúñiga, expresó esta convergencia cuando sintetizó la visión articulada del encuentro entre Fernández y López Obrador. En el texto, Zuñiga afirma que

\footnotetext{
Una alianza austral entre los dos gobiernos es apostar por el diálogo en busca de la distención regional para que las naciones de América Latina y el Caribe encuentren sus propias soluciones a los retos que enfrentan, en vez de contraponernos entre naciones hermanas y dinamitar los foros que deberían provocar la concertación (Zúñiga, 2019, s/p).
}

Los principales cuestionamientos que emergen de este escenario dicen respecto a las condiciones efectivamente existentes para la concretización de dicha propuesta. ¿Cuál es su potencial de éxito? ¿Cuáles son sus límites? En este texto, de hace un esfuerzo para contestar a tales cuestionamientos, articulando los contextos regionales y domésticos de los dos países. A partir del análisis del contexto en que ambos - Alberto Fernández y López Obrador - llegaron al poder, buscamos identificar los principales temas y retos que se les presenta para ambos en el ámbito internacional. De ahí, delineamos los alcances de esta iniciativa, que, en nuestra visión, están dados principalmente por los desafíos en la economía que ambos deben enfrentar.

Así, se trata de un trabajo que se regresa al examen de la coyuntura, esto es, de "un espacio de tiempo en el cual una combinación (o conjunción) específica de causas ejerce una influencia (causal o imaginativa) predominante en el curso de los eventos y en la producción de las ideas" (Rosenberg 2005, p. 42). Aunque este no sea el espacio para una digresión metodológica acerca de este tipo de ejercicio analítico ${ }^{4}$, es importante resaltar las limitaciones propias que él posee.

\footnotetext{
${ }^{4}$ Para eso, se recomienda la lectura de Cruz (2000), Rosenberg (2005) y Dobry (2014).
} 
Ya que se trata de un análisis de un proceso en curso, y por lo tanto sujeto a todo tipo de cambio, no intentamos que este texto comunique una interpretación consolidada del objeto, pero puede ofrecer una apreciación, a la luz de los hechos recientes, del horizonte que se presenta en la política exterior de Argentina y México y las posibilidades de articulación entre ambas.

\section{LA HERENCIA DEL GOBIERNO DE MAURICIO MACRI}

Mauricio Macri llegó a la presidencia de LA Argentina en diciembre de 2015, después de vencer al ex gobernador de Buenos Aires, Daniel Scioli, en segunda vuelta de las elecciones de aquel año. Heredero de una de las familias más ricas del país, y expresidente de uno de los más tradicionales clubes del país, el Boca Juniors, Macri ingresó en la política como jefe del Gobierno de Buenos Aires, y fue el principal fundador del Propuesta Republicana (PRO), partido por el cual disputó las elecciones.

Electo en medio de una ola de expectativas positivas, y emulando el slogan electoral de Barack Obama, "si, se puede", Macri basó su campaña más en la diferenciación con el kirchnerismo que propiamente en la discusión de un programa de gobierno. Aunque sin detallar sus propuestas, estaba claro que, si ganaba las elecciones, Macri haría un viraje en la política económica. Dicho cambio abandonaría el abordaje heterodoxo y voluntarista del último mandato de Cristina Kirchner para centrarse en un enfoque de corte ortodoxo y alineado a las expectativas del centro de la economía global.

Fue, pues, sin sorpresas, que se recibieron las primeras medidas económicas del gobierno de Macri, todavía en diciembre del 2015. En líneas generales, Macri adoptó un enfoque de fortalecimiento de la especialización productiva basada en la producción agraria, en la valorización de activos financieros y en una retracción del rol del Estado como inductor de la actividad económica (Vanoli, Cibils, Allami, 2018; Wahlberg, 2016). Bajo el argumento de que se trataba de reducir las ataduras a la libre iniciativa, y anclado a la premisa de que la confianza de los agentes económicos en el gobierno retraería los impactos negativos de la desregulación de los mercados, el gobierno generó un movimiento ambicioso en dirección a la liberalización económica, incluyendo la suspensión de la restricción a la venta de dólares, cambios en las retenciones de 
divisas del sector exportador, incremento en la tasa de interés, entre otras (NEFFA, 2017).

Esa agenda fue desarrollada en paralelo con una reorientación de política exterior, en el sentido de acercar a Argentina a los principales polos de poder occidental, principalmente los Estados Unidos y la Europa Occidental. En las pocas líneas dedicadas a la política exterior en su programa de gobierno, Macri propuso romper lo que calificaba como "aislamiento" de Argentina en el escenario internacional, a través de una normalización de la política exterior, que estaría al servicio de la realización del "potencial" del país (Cambiemos, 2015, s/p).

La primera demostración del viraje en la política exterior se dio con relación a Venezuela. Después de su asunción, Macri adoptó un tono muy duro y crítico hacia Nicolás Maduro, demandando la liberación de los presos políticos y presionando al Mercado Común del Sur (MERCOSUR) a suspender el país del bloque, lo que terminó ocurriendo en 2017, después de la deposición de la presidenta brasileña, Dilma Rousseff. Macri aun participó en los esfuerzos de creación del Grupo de Lima - y fue uno de los primeros Jefes de Estado en reconocer a Juan Guaidó como presidente interino, en enero de 2019.

Todavía, el foco de la política exterior permaneció direccionado a la búsqueda de apoyos para viabilizar la agenda económica del gobierno. En este sentido, la cuestión de la deuda externa fue el principal punto de articulación entre las agendas económica e internacional del gobierno de Macri - y dejó importantes consecuencias para el futuro de las relaciones exteriores del país. Dos semanas después de la asunción, el gobierno emitió casi 15 billones de dólares en deuda (Argentina, 2015) e inició un proceso de negociación con los llamados "fondos buitres" - los poseedores de títulos de la deuda que había sido puesta en moratoria en 2002 y que no participaron de las rodadas de reestructuración en 2005 y 2010.

En marzo de 2016, el gobierno aprobó en el Congreso - con apoyo de los congresistas de oposición - una ley que autorizaba el pago de los fondos buitres. Con la liquidación de los fondos buitres, el país salió de la situación de moratoria virtual que había iniciado en 2014, cuando una decisión de un juez de Nueva York congeló los 
depósitos del gobierno argentino para el pago de los acreedores que participaron de la reestructuración, hasta que hubiese la remuneración de los fondos buitres.

Sin restricciones de acceso a los mercados de capitales, e impulsado por entusiasmo del capital financiero con las credenciales favorables al mercado del gobierno, Argentina pasó por un rápido proceso de endeudamiento. En 2016, solamente dos años y medio después del inicio del gobierno de Macri, la deuda pública había crecido 83\%, llegando a cerca de 160 millones de dólares (Brenta, 2019, p. 185). Aunque haya sido sustentada por la retórica del financiamiento del sector productivo y de infraestructura, el principal destino de los recursos captados por la vía del endeudamiento fue la cobertura de déficits provocados por la reducción de impuestos a los sectores rentistas y al agronegocio, y, posteriormente, el financiamiento de la fuga de capitales (Vanoli, Cibilis, Allami, 2018; Brenta, 2019).

De acuerdo con Brenta (2019), la inversión en infraestructura como justificación para el endeudamiento externo retomaba las recomendaciones emitidas por los organismos multilaterales, como el Banco Mundial y el Banco Interamericano de Desarrollo, en medio de la crisis de la Zona del Euro y del anuncio de la Belt and Road Iniciative, por parte de China. Así, para la autora, la emisión de la deuda para el financiamiento en infraestructura debe ser enmarcado en la "puja por mantener y profundizar la globalización bajo la hegemonía occidental, estrategia impulsada por los países avanzados y sus corporaciones en circunstancias y tiempos precisos" (Brenta, 2019, p. 187).

El año de 2018 fue un momento crítico de la trayectoria del endeudamiento de la Argentina. El incremento de las tasas de interés en los Estados Unidos y el alza de los precios del petróleo, sumados al deterioro fiscal, iniciaron una intensa salida de capitales y la consecuente caída de las reservas internacionales del Banco Central. Frente a esto, y con apoyo explícito del gobierno de Estados Unidos (The White House, 2018; U.S Department of Treasury, 2018), Argentina dio inicio a las negociaciones con el Fondo Monetario Internacional (FMI), que resultaron en un acuerdo standby en junio de 2018. 
En una muestra de respaldo a la política económica del gobierno de Macri, el FMI acordó un préstamo de US\$50 billones - el mayor préstamo realizado por este organismo. El valor, equivalente a $1.110 \%$ de la cuota del país en el FMI, fue justificado por el organismo con base en el apoyo a la "transformación sistémica" de la economía, en la cual el país estaba comprometido desde 2015 (International Monetary Fund, 2019). En cambio, el país reafirmaba su compromiso con las reformas liberales y se comprometía a reverter el déficit fiscal hasta 2021.

Con una firme red de apoyos externos, el deterioro de la economía no se detuvo, enredado en sus propias contradicciones. En 2019, Macri, que siendo candidato había prometido "pobreza cero", se tornó presidente de un país en que 35,4\% de la población se encontraba en situación de pobreza y la indigencia afectaba a cerca de 3.3 millones de personas (INDEC, 2019). La inflación - una vieja llaga de la economía argentina alcanzaba 53,3\%, al paso en que el peso argentino perdió $68 \%$ de su valor frente al dólar, y el porcentaje de trabajadores desempleados y aquellos en empleos precarios o informales superaba $50 \%$ de la población económicamente activa del país (Banco Central de la República Argentina, 2019; INDEC, 2019).

\subsection{El Contexto Electoral}

Con la situación económica abiertamente desfavorable a su proyecto de reelección, Mauricio Macri buscó repetir la estrategia que había sido exitosa cuatro años antes, explorando la polarización política de Argentina como mecanismo para mitigar los impactos de la crisis económica en el debate electoral. El blanco preferencial de Macri era la senadora y expresidenta, Cristina Fernández de Kirchner.

Principal figura de la oposición del país, Cristina Kirchner vivía una situación peculiar, producida por el hecho de ser, simultáneamente, el nombre más competitivo para enfrentar a Macri en una primera ronda, pero cuyos altos índices de rechazo podrían llevar a la derrota en la segunda vuelta (La Nación, 2019). Su nombre era igualmente rechazado por sectores del peronismo, lo que limitaba la formación de un consenso entre las fuerzas de este campo político. La síntesis de esta contradicción fue descrita de manera precisa por el propio Alberto Fernández, al declarar que "con Cristina no alcanza y sin ella no se puede" (2018, p. 12). 
El embrollo del peronismo fue encerrado por medio de un movimiento sorprendente. El 18 de mayo, Kirchner divulgó un video en que anunciaba que, contrariamente a todas las expectativas gestadas hasta entonces, sería candidata a vicepresidenta, en una fórmula liderada por Alberto Fernández. El hecho de ser Fernández el candidato a presidente fue tan espantoso como la propia renuncia a la cabeza de placa en sí, por el histórico de conflictos y críticas públicas entre ellos.

Aunque sorprendente, el arreglo fue exitoso desde el punto de vista electoral. Alberto Fernández consiguió aglutinar a los principales sectores del peronismo alrededor de su nombre, retirando candidaturas alternativas, especialmente la de Sergio Massa. Al mismo tiempo, la estrategia del gobierno de apostar por la polarización para desviar el debate de las cuestiones económicas se quedó comprometida. Una tercera, y decisiva consecuencia, inviabilizar la candidatura de Roberto Lavagna, el ministro de economía en el gobierno de Néstor Kirchner que venía buscando consolidarse como alternativa a la polarización entre Macri y Cristina Kirchner.

Con el peronismo unificado, y en medio del fracaso económico del gobierno de Macri, la fórmula Alberto Fernández-Cristina Kirchner obtuvo una expresiva victoria en las elecciones primarias, realizadas en agosto. Dos meses después, el 27 de octubre de 2019, la fórmula recibiría 48\% de los votos válidos, venciendo en primera vuelta.

\section{EL GOBIERNO DE ENRIQUE PEÑA NIETO EN MÉXICO}

La llegada de Andrés Manuel López Obrador (AMLO) a la presidencia de México representó un cambio histórico en la política del país. El Movimiento Regeneración Nacional (Morena) asumió por primera vez el poder Ejecutivo, después de ser ocupado 77 años por el Partido Revolucionario Institucional (PRI), entre 1929 y 2000 y entre 2012 y 2018, y 12 años del Partido Acción Nacional (PAN), entre 2000 y 2012.

Múltiples factores contribuyeron al desgaste del gobierno de Enrique Peña Nieto (2012-2018). El lanzamiento del “Pacto por México, el 2 de diciembre de 2012, generó expectativas de crecimiento económico rápido y sostenido. El acuerdo fue negociado con los principales líderes políticos de oposición, el PAN y el $\mathrm{PRD}^{5}$, en aquel momento presidido por López Obrador. El objetivo era promover los cambios necesarios para

\footnotetext{
${ }^{5}$ Partido de la Revolución Democrática.
} 
consolidar la transición democrática en México y fomentar el crecimiento económico (Embajada de México en Italia, 2012). Sin embargo, desacuerdos en torno a la reforma energética en el interior del PRD llevaron a la salida de López Obrador del partido y a la creación del Morena, en 2014.

Contrario a las expectativas del gobierno, las reformas tuvieron efectos limitados. El Producto Interno Bruto (PIB) de México, entre 2013 y 2018, creció en promedio 2,4\% al año (Organization for Economic Co-operation and Development, 2020). También contribuyeron al desgaste del gobierno las denuncias de corrupción que involucraron a Angélica Rivera, esposa del presidente, y también de aliados cercanos, cuyo caso más emblemático fue el de Javier Duarte de Ochoa, gobernador de Veracruz por el PRI. Además, hay que mencionar la violencia extrema, en 2018, con 36.685 asesinatos, así como la desaparición forzada de 43 estudiantes en el estado de Guerrero, el 26 de septiembre de 2014 (Instituto Nacional de Estadística y Geografía, 2020).

Además de las problemáticas nacionales, el gobierno del PRI enfrentó incertidumbres considerables en el sistema internacional. La elección de Donald Trump, en 2016, comprometió la estabilidad política y económica interna cuando el entonces candidato amenazó con salir del Tratado de Libre Comercio de América del Norte (NAFTA, en inglés). El 19 de enero de 2017 y el 19 de julio de 2017, primer semestre del gobierno de Trump, el peso mexicano se devaluó 21\% (Banco de México, 2020). Aunque las relaciones económicas bilaterales han sido históricamente intensas, el NAFTA institucionalizó la dependencia de México con los Estados Unidos y tornó más complejo el establecimiento de contrapesas. En 2018, los EE.UU. fueron el destino de 79\% de las exportaciones y origen de $46 \%$ de las importaciones de México (Secretaria de Economía, 2020a). El vecino también fue el principal origen de las Inversiones Extranjeras Directas en México, 35\% del total, en 2019 (Secretaría de Economía, 2020b).

La presión de los empresarios y de los estados de la frontera con México pueden haber sido elementos relevantes para que Trump adoptara la posición de negociar la modernización del NAFTA, con inicio de la primera ronda en agosto de 2017. La intención del gobierno de México era no extenderse demasiado, por el inicio del 
calendario electoral en el país. El transcurso de las negociaciones limitaba una posición más asertiva del gobierno de Enrique Peña Nieto. Así, el gobierno de México tuvo que adoptar una posición defensiva y de contención de daños (González; Castillo, 2019), la cual generó desgastes frente a la opinión pública. El Tratado México-Estados UnidosCanadá (USMCA, en inglés) fue firmado el 30 de noviembre de 2018, en vísperas de toma de posesión de López Obrador, que tendría lugar el 2 de diciembre de 2018.

La crisis en la relación con los Estados Unidos intensificó los debates de cómo disminuir la dependencia con el país vecino (González; Castillo, 2019). América Latina y el Caribe serían regiones importantes en una estrategia de diversificación de las relaciones económicas, por sus vínculos históricos y culturales con México. Sin embargo, la región representa poco en las exportaciones e importaciones del país. En 2018, los miembros de la Asociación Latinoamericana de Integración (ALADI) fueron destino de solo $3,4 \%$ de las exportaciones mexicanas y $2,5 \%$ de las importaciones (Secretaría de Economía, 2020a). El gobierno de Peña Nieto no quiso apostar a esta política por temor al compromiso de las negociaciones para modernizar el NAFTA (González; Castillo, 2019).

María Elena López Montero y Carlos Contreras Romero (2010) indican que la diversificación de las relaciones económicas internacionales aparece en el discurso diplomático del país como una forma de conquistar mayor autonomía. Ella parte del presupuesto que la "doble posición geopolítica" de México es una oportunidad (González, 2006). El país está integrado económicamente al norte, pero tiene vínculos sociales, culturales e históricos con la América Latina y el Caribe. Sin embargo, ninguna región es capaz de equilibrar las relaciones económicas internacionales de México (Montero; Romero, 2010). Así, aparte de la aparente crisis en las relaciones con los Estados Unidos, América Latina no representaba de hecho una alternativa.

El conjunto de esos elementos contribuyó al desgaste del gobierno de Enrique Peña Nieto, quien dejó la presidencia con solo 26\% de aprobación (Buendía \& Laredo, 2018). López Obrador venció las elecciones con 53,19\% de los votos válidos (Instituto Nacional Electoral, 2018). Él daba señales de continuidad en la política exterior: los Estados 
Unidos serían prioridad y América Latina y el Caribe tendrían poco espacio en la inserción internacional de México.

\section{LA POLÍTICA EXTERIOR DE FERNÁNDEZ Y OBRADOR Y LAS RELACIONES CON AMÉRICA LATINA}

\subsection{Los retos de la política exterior del gobierno de Alberto Fernández}

Darle a la política exterior un abordaje progresista fue uno de los objetivos explícitamente anunciado por Alberto Fernández, anclado en la crítica al modelo económico del gobierno de Macri. En esa visión, el gobierno de Macri tendría hecho una política exterior basada en la lectura equivocada del escenario internacional, resumiendo la "vuelta al mundo" en el acceso a los mercados internacionales de crédito. En su plataforma electoral, la "Frente de Todos" diagnosticó la existencia de tensiones resultantes de la transición de un orden unipolar hacia otro multipolar, bien como de un escenario de desintegración regional, que "contribuyen a una creciente intrascendencia de la región a nivel global que agudiza la dependencia y la pérdida de autonomía" (Frente de Todos, 2019, s/p).

Durante la campaña, Fernández se encontró con líderes importantes de la izquierda latinoamericana, como el ex presidente de Uruguay, José Mujica, además de visitar en la prisión al expresidente brasileño, Lula da Silva. Además de estos gestos, el propio hecho de tener a Cristina Kirchner como vicepresidenta secundaba las expectativas de que su gobierno plantearía una política exterior de refuerzo de una agenda progresista, como contrapunto a las políticas neoliberales y más orientada a la autonomía nacional y regional.

En términos prácticos, ese "progresismo" puede ser sintetizado en tres aspectos principales: a) retomaba a la autonomía como idea-fuerza de la política exterior; b) revitalizaba los mecanismos de concertación regional gestionados en la década pasada; c) tomaba impulso del Grupo de Puebla en la mediación de la crisis venezolana. Sin embargo, hay que entender su significado concreto en la política exterior de Argentina a la luz de las circunstancias objetivas en medio a las cuales el gobierno conducirá las relaciones exteriores del país. 
El primer elemento a ser considerado es la crisis económica, sobre todo el problema de la deuda externa - que el programa electoral de Fernández reconocía como una restricción a la política exterior, que expone al país a un "sistema de poderes e intereses concentrados que intentarán restar autonomía y grados de libertad a nuestras decisiones soberana" (Frente de Todos, 2019, s/p). En 2019, Argentina registró una caída del 3\% del PIB (CEPAL, 2019, p. 2) y el progresivo deterioro del comercio con los países del MERCOSUR - que constituyen los principales destinos de las exportaciones de mayor valor agregado. La deuda - cuya dinámica sintetizamos anteriormente implicará en la necesidad de negociaciones con el FMI, y su éxito será determinado no solamente por las relaciones directas con el Fondo, pero también por la dinámica establecida junto al gobierno de los Estados Unidos.

El panorama político de la región es otro frente del problema. Por más intenso que sea el activismo argentino, es poco probable que la dinámica del regionalismo suramericano sea significativamente alterada en cuanto a permanecer estable su actual correlación de fuerzas. Brasil representa un reto particular, porque, además de la franca animosidad establecida entre Fernández y Jair Bolsonaro, la política exterior brasileña se encuentra en abierta contradicción con las directrices en abierta contradicción a las directrices consolidadas a lo largo de las últimas décadas, y que fueron responsables del proceso de construcción de confianza y organización de la sociedad estratégica con Argentina. La densidad de las relaciones bilaterales impide, sin embargo, que se adopte una posición de desatención y exige un delicado ejercicio de construcción de agendas positivas, especialmente en el ámbito del MERCOSUR.

Finalmente, un aspecto a ser considerado es la relación entre Fernández y Cristina Kirchner. De hecho, esta es una gran incógnita, ya que los poderes usualmente disfrutados por la vicepresidencia argentina son incompatibles con el peso político de Cristina Kirchner. En el caso específico de la política exterior, la expresidenta puede tener un rol importante, especialmente en las relaciones bilaterales con Rusia, Venezuela y China, países con los que ella desarrolló importantes agendas cuando fue presidenta. 
¿Qué podemos desprender de ese panorama? A partir de una evaluación preliminar, y sin intención de ser predictivos, el cuadro que se presenta a Alberto Fernández en el campo de la política exterior se asemeja al que él debe enfrentar internamente: la necesidad de construir equilibrios y apaciguar tensiones. Los discursos y los pronunciamientos de Fernández sugieren que él está consciente de ello. En su discurso de asunción, por ejemplo, dijo que desea una relación de cooperación con los acreedores, y resolver "el problema de una deuda insostenible que hoy tiene Argentina no es una cuestión de ganarle una disputa a nadie" (Fernández 2019, s/p). Respecto a Brasil, destacó la importancia de la cooperación entre los países, más allá de "cualquier diferencia personal de quienes gobiernan la coyuntura" (Fernández 2019, s/p). En cuanto a Venezuela, aunque tenga alejado de la retórica combativa del gobierno de Macri, Fernández eligió permanecer simultáneamente en el Grupo de Lima y en el de Puebla.

\subsection{La política exterior del gobierno de Obrador para América Latina}

La política exterior no fue un tema de mucha relevancia en la campaña presidencial de México. Fue debido a las amenazas del presidente Trump y a las negociaciones para la modernización del NAFTA que las relaciones con el vecino recibieron atención. Aparte de reconocer la dependencia, López Obrador no mencionó la diversificación como una de sus propuestas. La América Latina no fue recordada y el Caribe apenas en política migratoria.

La posición de López Obrador fue contraria a las especulaciones hechas en la campaña electoral de que el nuevo gobierno de México pudiera tener una política exterior hacia la región, por ser considerado un candidato progresista, y por la coyuntura de crisis en las relaciones con Estados Unidos. La visita de Alberto Fernández, después de ser electo, a México, en noviembre de 2019, contribuyó a alimentar especulaciones de que los dos formasen un bloque progresista en América Latina para hacer frente a los gobiernos más de derecha, como los de Chile y Brasil.

El Plan Nacional de Desarrollo 2019-2024 y las políticas adoptadas en el primer año de gobierno no indicaron un nuevo rol de América Latina y el Caribe en la política exterior de México, sobre todo después del USMCA. El gobierno de AMLO señaló que 
aprovecharía las relaciones con los Estados Unidos para desarrollar su agenda interior. Así, en el período de sucesión, el gobierno participó de las negociaciones para la modernización del NAFTA. Jesús Seade ${ }^{6}$ fue nombrado representante del gobierno de transición para acompañar las negociaciones, lo que señaló apoyo del próximo presidente de México al Tratado y redujo especulaciones de posibles rupturas.

La política exterior en general, y no solamente las relaciones con América Latina, fue tratada de manera poco detallada. Cuando fue cuestionado acerca de su política exterior, AMLO contestó que "la mejor política exterior es la interior", lo que indica la priorización a la agenda doméstica. En su primer año de gobierno, él no realizó ningún viaje internacional. En la Cumbre del G-20, en junio de 2019, y en visita a China, en julio del mismo año, el Secretario de Relaciones Exteriores, Marcelo Ebrard, representó al gobierno mexicano.

Según el Plan Nacional de Desarrollo 2019-2024, el principal objetivo de la política exterior de México es recuperar sus principios históricos, los cuales sean: no intervención, autodeterminación, cooperación para el desarrollo, solución pacífica de controversias y respeto a los derechos humanos (Diario Oficial de la Federación, 2019). América del Norte es prioridad, posición justificada por el "intenso intercambio económico, cultural y demográfico" (Diario Oficial de la Federación, 2019). Además de subrayar la participación de México en el NAFTA, el documento también clasifica como un tema relevante la cooperación en la cuestión migratoria, que moviliza parte de los países de la América Central.

Con respecto a América Latina, se dice que se necesario "impulsar con énfasis" la integración latinoamericana, pero no se menciona ninguna iniciativa en la que México participa, como la CELAC y la Alianza del Pacífico (Diario Oficial de la Federación, 2019). El Plan tampoco hace ninguna mención a China, lo que genera incertidumbre sobre el futuro de la Cumbre China-CELAC, aunque la realización de un foro de secretarios aparezca como uno de los objetivos de la presidencia de México en la CELAC, desde enero de 2020.

\footnotetext{
${ }^{6}$ Subsecretario para América del Norte de la Secretaría de Relaciones Exteriores.
} 
Aunque los principales temas de la política exterior de México también son de interés para otros países de América Latina y el Caribe, como migración, comercio y seguridad, el gobierno de México prefiere negociar bilateralmente con los Estados Unidos. En el ámbito del comercio y de la seguridad, los dos países tienen acuerdos, el USMCA y la Iniciativa Mérida. Además, esos temas tienen fuerte interlocución con la agenda interior de México, lo que justifica la preferencia por la vía bilateral.

La migración fue el tema central de la política exterior en el primer año de gobierno de López Obrador. El cambio en los flujos desde 2007, reforzaron el rol del gobierno de México para contener la inmigración indocumentada. Desde entonces, los mexicanos no son los que más migran sin documentación. Así, México se volvió un territorio de tránsito para inmigrantes de otras nacionalidades hacia Estados Unidos. (Pew Research Institute, 2019).

En 25 de enero de 2019, el gobierno de México y de los Estados Unidos acordaron una medida de control del flujo migratorio. El Protocolo de Protección, popularmente conocido como "Permanecer en México", obliga a los migrantes que llegan a Estados Unidos sin documentación a esperar en México por la decisión de la corte de EE.UU.. Entre el 29 de enero de 2019 y el 11 de julio de 2019, el Instituto Nacional de Migración del Gobierno de México (2019) recibió casi 20 mil solicitudes de extranjeros de América Latina y el Caribe que solicitaban asilo en Estados Unidos. Según relatos obtenidos en Tijuana, muchos de los que están en esas condiciones recibieron visa humanitaria del gobierno de México, que les permite trabajar en el país. Los que no tienen la visa están en condiciones ilegales o fueron deportados a sus países de origen.

El Plan fue renovado, en junio de 2019, después de amenazas del gobierno de Trump de que asignaría impuestos del 5\% a las importaciones de México. Como parte del acuerdo, el gobierno de López Obrador envió efectivos de la Guardia Nacional a la frontera sur de México. Ésta es una señal de endurecimiento de la política migratoria de Estados Unidos, en colaboración con el gobierno de México. También es señal de que López Obrador no se dispone a defender a los centroamericanos si eso implica un conflicto con el gobierno de Trump. Con las críticas, en junio de 2019, el gobierno de México lanzó el Plan de Desarrollo Integral El Salvador - Guatemala - Honduras - México, 
con participación de la Comisión Económica para América Latina y el Caribe (CEPAL). Su objetivo es combatir la migración internacional de manera multidimensional, desde su origen, tránsito, destino y retorno (Naciones Unidas, 2019). Además de asistencia consular, es necesario promover el "desarrollo productivo" en América Central, que sea capaz de fomentar la producción local, facilitar las relaciones comerciales y desarrollar proyectos de infraestructura, como el Tren Maya ${ }^{7}$ y el Corredor Transístmico ${ }^{8}$ (Diario Oficial de la Federación, 2019).

El gobierno de López Obrador reconoció no tener los recursos necesarios para la implantación del Plan. Así, la puesta en marcha de dicho Plan depende de la ONU y de los socios externos, como Alemania, España, Chile y los países del G-20. Aunque el Plan tiene una perspectiva más humanitaria en comparación con programas anteriores, el fomento de las actividades productivas en América Central no es novedad. El Plan Puebla Panamá, de 2002, y el Proyecto Mesoamérica, de 2008, también tenían el desarrollo como uno de sus principales objetivos.

Las medidas militares en la frontera norte pudieron detener la imposición de aranceles por parte de Estados Unidos, lo que generaría graves perjuicios económicos a México. Con todo, dichas medidas también sirvieron para apaciguar posiciones antimigrantes en el interior de México. Según la encuesta de Washington Post y del periódico Reforma (2019), 59\% de los mexicanos tenían opiniones favorables hacia el acuerdo migratorio con Estados Unidos y 51\% estaba de acuerdo en la utilización de la Guardia Nacional para combatir el flujo indocumentado.

Aunque coopere con los Estados Unidos en migración, la posición de la Secretaría de Relaciones Exteriores con relación a las elecciones en Venezuela contradijo la del gobierno de EE.UU. y de otros países de América Latina, lo que puede indicar relativo protagonismo de la política exterior de López Obrador. El gobierno de México no reconoció el auto proclamado presidente de Venezuela, Juan Guaidó. En un comunicado de prensa, la Secretaría de Relaciones Exteriores de México (2019a) declaró que fue una posición neutral y alienada a los principios de política exterior del país. El comunicado defendió una posición negociada para el conflicto, con

\footnotetext{
${ }^{7}$ Ferrocarril que cruza la Península de Yucatán.

${ }^{8}$ Canal interoceánico que conecta los puertos de Oaxaca y Veracruz.
} 
participación de la ONU y de Uruguay, que adoptó posición semejante a la del gobierno de México.

Por fin, el gobierno de México dio asilo al ex presidente de Bolivia, Evo Morales, en noviembre de 2019. La Secretaría de Relaciones Exteriores (2019b) caracterizó como golpe los acontecimientos que llevaron a la renuncia de Evo Morales y pidieron respeto a la democracia en Bolivia. Así como en Venezuela, el gobierno mexicano pidió una solución negociada, con apoyo de la ONU. Morales viajó en un avión de la Fuerza Aérea Mexicana y permaneció en México por cerca de un mes, cuando fue a Argentina como refugiado.

La posición del gobierno de México con relación a la coyuntura en Venezuela y en Bolivia indicó una tentativa de recuperar los principios históricos de la política exterior, además de adoptar una posición de liderazgo en la negociación multilateral de los conflictos regionales. Con todo, aparte de haber solicitado apoyo a la ONU en ambas ocasiones, el gobierno no llevó el tema a la CELAC, lo que indica baja prioridad a América Latina. En los dos casos, el posicionamiento puede ser entendido a la luz del voto contrario de la delegación de México a la expulsión de Cuba de la Organización de los Estados Americanos (OEA), en 1962. Según Mario Ojeda (2011), la posición con relación a Cuba partió de un entendimiento previo con los Estados Unidos que, por la importancia estratégica de la estabilidad de México, toleraban posiciones disidentes de la política exterior de México que favoreciesen el consenso interno en el país.

En ese contexto, la posición del gobierno de Andrés Manuel López Obrador señaliza a sus bases progresistas, que defienden políticas más independientes con relación a los Estados Unidos. El país también tiene un amplio histórico de concesión de asilo, cuyos casos más emblemáticos son el de León Trotsky (1937) y Fidel Castro (1955). Con todo, el gobierno de México no amenazó las relaciones con los Estados Unidos, a pesar de las divergencias políticas. Eso indica que hay un status quo aceptado por los dos países en la condición de las relaciones bilaterales, cuando está en juego la estabilidad interna en México. 


\section{CONSIDERACIONES FINALES}

La elección de Alberto Fernández, en Argentina, y de Andrés Manuel López Obrador, en México, generó grandes expectativas de que los dos pudiesen liderar un frente regional progresista en América Latina, en contraposición a los gobiernos más identificados con una orientación espectros política de derecha en el continente, el caso de Chile y de Brasil. La visita de Fernández a México, la primera que hizo al exterior después de ser electo, tenía como objetivo impulsar la agenda bilateral entre los países y discutir los rumbos de la política y de la economía en América Latina. En ese contexto, el artículo analizó elementos locales, regionales e internacionales que posibilitan o limitan esa agenda por parte de Argentina y México.

Argumentamos que los presidentes llegaron al poder en medio de una coyuntura política y económica compleja en las relaciones interamericanas. La CELAC, principal mecanismo multilateral, se encuentra vacía. Aunque los gobiernos de Argentina y México sean miembros, el gobierno de Brasil suspendió su participación en enero de 2020, lo que limitó aún más el rol del órgano en la política regional y en las relaciones con socios externos, como en las cumbres con China y la Unión Europea. Las nuevas prioridades de la política exterior brasileña hicieron las relaciones bilaterales un reto para el gobierno de Fernández, además de la propia coyuntura económica doméstica.

Aunque Fernández exprese más abiertamente la adopción de una política exterior progresista, los desafíos que se presentan a las relaciones exteriores de Argentina imponen un límite a las iniciativas que se pueda desarrollar desde este país. El tema de la deuda externa es el reto más significativo en este contexto, pues requiere un manejo cuidadoso de las relaciones con el FMI y el gobierno de Estados Unidos. Po otro lado, López Obrador es más cauteloso en lo que se refiere a la posibilidad de coordinar la agenda bilateral y regional para obtener más autonomía en el sistema internacional. México no tiene, en el corto y mediano plazo, alternativa a la dependencia económica en relación con los Estados Unidos, lo que llevó a López Obrador a participar en las negociaciones para modernizar el NAFTA y a cooperar en el control del flujo migratorio en América Central. Así, como discutimos, el gobierno de López Obrador expuso un 
interés muy limitado en las relaciones con América Latina. Eso desarma tentativas más sustantivas de cooperación con el gobierno de Argentina.

Lo que se puede inferir de este cuadro es que, por ahora, la propuesta de un activismo en torno a una agenda progresiva deberá estar más restringida a la retórica que a la articulación de iniciativas sustantivas. Esto, por supuesto, no es una profecía, sino una tendencia que extraemos del examen de la situación que se presenta a ambos países. La complejidad del contexto regional, las diferencias entre las agendas de política exterior de los dos gobiernos y los retos domésticos que deben contornar son obstáculos que, aunque no sean insuperables, traen consigo costos que, a nuestro ver, son más altos que los beneficios que puede generar en lo corto plazo.

\section{REFERENCIAS BIBLIOGRÁFICAS}

Argentina, Secretaría de Hacienda y Secretaría de Finanzas. (2015). Resolución Conjunta 7/2015 $y$ 3/2015. Disponible en: <https://www.boletinoficial.gob.ar/detalleAviso/primera/138789/20151223>. [Aceso en: 01 marzo 2020].

Banco Central de la República Argentina. (2019). Principales variables. Disponible en: <http://www.bcra.gov.ar>. [Acceso en: 01 marzo 2020].

Banco de México. (2020). Sistema de Información Económica: Mercado Cambiario (Tipos de Cambio). Disponible en: <https://www.banxico.org.mx/tipcamb/main.do?page=tip\&idioma=sp>. [Acceso en: 31 enero 2020].

Brenta, N. (2019) Historia de la deuda externa argentina: de Martinez de Hoz a Macri. Buenos Aires: Capital Intelectual.

Buendía \& Laredo. (2018). Aprobación presidencial. Disponible en: <http://buendiaylaredo.com/publicaciones/444/APROBACION.pdf>. [Acceso en 31 enero 2020].

CEPAL. (2019). Preliminary Overview of the Economies of Latin America and the Caribbean: Argentina. Disponible en: <https://repositorio.cepal.org/bitstream/handle/11362/45001/BPI2019_Argentina_en. pdf?sequence=128\&isAllowed=y>. [Acceso en: 01 marzo 2020].

Cruz, S. V. (2000) 'Teoria e Método na Análise de Conjuntura', Educação \& Sociedade, ano $X X I$, n. 72.

Diario Oficial de la Federación. (2019). Plan Nacional de Desarrollo 2019-2024, 12 de julho.

Disponible

en: 
<https://www.dof.gob.mx/nota_detalle.php?codigo=5565599\&fecha=12/07/2019>. [Acceso en 31 enero 2020].

Dinatale, M. (2015) Alberto Fernández propone una política exterior progresista, pero sin enfrentarse a Donald Trump. Disponible en: <https://www.infobae.com/politica/2019/11/05/alberto-fernandez-propone-unapolitica-exterior-progresista-pero-sin-enfrentarse-a-donald-trump/>. [Acceso en: 01 marzo 2020].

Dobry, M. (2014) Sociologia das crises políticas. São Paulo: Editora Unesp.

Embajada de México en Itália. (2012). Pacto por México. Disponible en: <https://embamex.sre.gob.mx/bolivia/images/pdf/REFORMAS/pacto_por_mexico.pdf >. [Acceso en: 30 enero 2020].

Fernández, A. (2019) Palabras del presidente Alberto Fernández en su acto de asunción ante la Asamblea Legislativa. Disponible en: $<$ https://www.casarosada.gob.ar/informacion/discursos/46596-palabras-delpresidente-alberto-fernandez-en-su-acto-de-asuncion-ante-la-asamblea-legislativa>. [Acceso en: 01 marzo 2020].

González, G. G. (2006) 'México ante América Latina: Mirando de reojo a Estados Unidos', in: Schiavon, J. A.; Spencer, D.; Oliveira, M. V. (eds.), En busca de una nación soberana: Relaciones internacionales de México, siglos XIX y XX. México, D.F.: Centro de Investigación y Docencia Económicas/Secretaria de Relaciones Exteriores, pp. 463-508.

.; Castillo, R. M. (2019) 'La política exterior del gobierno de Enrique Peña Nieto hacia América Latina y el Caribe en un mundo en transición: una trama de tres actos', Foro Internacional, LIX, 3-4, pp. 763-808, [online]. Disponible en: $<$ https://forointernacional.colmex.mx/index.php/fi/article/view/2640>. [Acceso en: 4 febrero 2020].

Instituto Nacional de Estadística y Geografía. (2020). Mortalidad. Conjunto de datos: Defunciones por homicidios. Disponible en: $<$ https://www.inegi.org.mx/sistemas/olap/consulta/general_ver4/MDXQueryDatos.asp? proy $=>$. [Acceso en 30 enero 2020].

Instituto Nacional Electoral. (2018). Cómputos Distritales 2018: Elecciones Federales. Disponible en: <https://computos2018.ine.mx/\#/presidencia/ nacional/1/1/1/1>. [Acceso en 14 febrero 2020].

International Monetary Fund. (2018). IMF Executive Board Approves US\$50 Billion Stand-By Arrangement for Argentina. Disponible en: <https://www.imf.org/en/News/Articles/2018/06/20/pr18245-argentina-imf-executiveboard-approves-us50-billion-stand-by-arrangement>. [Acceso en: 01 marzo 2020].

INDEC. (2019). Informe Estadístico. Disponible en: <https://www.indec.gob.ar>. [Acceso en: 01 marzo 2020]. 
La Nación. (2019). El PJ con menos rechazo que el kirchnerismo. Disponible en: <https://www.lanacion.com.ar/politica/el-pj-con-menos-rechazo-que-el-kirchnerismonid2202927>. [Aceso en: 01 marzo 2020].

Montero, M. E. L.; Romero, C. C. (2010). 'El mito de la diversificación de las relaciones exteriores de México: las relaciones con Europa, Asia-Pacifico y Medio Oriente', in: Montero, M. E. L. (coord.), Temas y escenarios en la agenda de política exterior de los gobiernos de la alternancia política en México. México, D.F.: DGAPA, pp. 229-256.

Naciones Unidas. (2019). Disponible en: <https://www.gob.mx/cms/uploads/attachment/file/462720/34.Hacia_un_nuevo_estilo _de_desarrollo__Plan_de_Desarrollo_Integral_El.pdf>. [Acceso en: 13 febrero 2020].

Neffa, J. (2017) 'El contexto socioeconómico argentino actual'. Cuadernos del Cendes, 4(95). Caracas.

Observatório de Política Exterior Argentina. (2019). Informe de política exterior argentina, n. 542. Disponible en: <https://gedes-unesp.org/wpcontent/uploads/2019/11/OPEA-542.pdf>. [Acceso en: 01 marzo 2020a].

(2019). Informe de política exterior argentina, n. 543. Disponible en: <https://gedes-unesp.org/wp-content/uploads/2019/11/OPEA-543.pdf>. [Acceso en: 01 marzo 2020b].

Ojeda, M. (2011). Alcances y límites de la política exterior de México. Ciudad de México: El Colegio de México.

Organization for Economic Co-operation and Development. (2020). Data: Real GDP forecast. Disponible en: <https://data.oecd.org/gdp/real-gdp-forecast.htm>. [Acceso en: 30 enero 2020].

Página 12. (2018). Con Cristina no alcanza y sin ella no se puede. Disponible en: <https://www.pagina12.com.ar/94244-con-cristina-no-alcanza-y-sin-ella-no-se-puede>. [Acceso en: 01 marzo 2020b].

Panizza, F. (2006). 'La Marea Rosa', Observatório Político Sul-Americano: Análise de Conjuntura. n.8, p. 1-16.

Pew Research Institute. (2019). Disponible en: <https://www.pewresearch.org/facttank/2019/06/12/us-unauthorized-immigrant-population-2017>. [Acceso en: 13 febrero 2020].

Rosenberg, J. (2003) 'Globalization Theory: a post mortem', International Politics, n. 42, p. 2-74. [Acceso en: 01 marzo 2020].

Secretaría de Economía. (2020a). Comercio Exterior / Estadísticas Históricas. Disponible en: $\quad<$ https://www.gob.mx/se/acciones-y-programas/comercio-exterior-estadisticashistoricas?state=published $>$. [Acceso en: 30 enero 2020]. 
(2020b). Información estadística de la Inversión Extranjera Directa: Datos y Recursos. Disponible en: <https://datos.gob.mx/busca/dataset/informacion-estadisticade-la-inversion-extranjera-directa>. [Acceso en: 30 enero 2020].

Secretaria de Relaciones Exteriores. (2019a). Mexico Following Its Constitutional Principles; Supports United Nations Appeal on Venezuela, Press Release 012. Disponible en: $\quad<$ https://www.gob.mx/sre/prensa/mexico-following-its-constitutional-principlessupports-united-nations-appeal>. [Acceso en: 14 febrero 2020].

(2019b). Postura del Gobierno de México sobre los acontecimientos en Bolivia, Comunicado No 396. Disponible en: <https://www.gob.mx/sre/prensa/postura-delgobierno-de-mexico-sobre-los-acontecimientos-en-bolivia>. [Acceso en: 14 febrero 2020].

The White House. (2018). Statement from the Press Secretary on Argentina. Disponible en: $\quad<$ https://www.whitehouse.gov/briefings-statements/statement-press-secretaryargentina>. [Acceso en: 9 marzo 2020].

United States Department of Treasury. (2018). Readout from a U.S. Treasury Spokesperson on Under Secretary of the Treasury David Malpass' Meeting with Minister of the Treasury Nicolas Dujovne of Argentina. Disponible en: $<$ https://home.treasury.gov/news/press-releases/sm0384>. [Acceso en: 01 marzo 2020].

Vanoli, A.; Cibils, A.; Allami, C. (2018). Los cambios en la era Cambiemos: Adiós producción y consumo, hola (otra vez) especulación financiera. Análisis, n.36. Friedrich Ebert Stiftung Argentina.

Wahlberg, F. (2016). 'Cambiemos menos industria por más deuda', in: ¿A dónde va la economía del gobierno de Macri? Documento de Trabajo de Economistas de Izquierda (EDI) y la Oficina de Enlace Buenos Aires de la Rosa Luxemburgo.

Washington Post-Reforma. (2019). Disponible en: <https://gamescdn.washingtonpost.com/notes/prod/default/documents/9a99d581-eeda-47eb-b2090943d1ed1866/note/a67de03f-e710-4a05-a2e9-1eb5f9c62bd0.pdf\#page=1>. [Acceso en: 14 enero 2020].

Zúñiga, M. (2019) México y Argentina, oportunidad para una alianza austral. La Jornada, 04 nov. Disponible en: <https://www.jornada.com.mx/2019/11/04/politica/014a1pol>. [Acceso en: 01 marzo 2020]. 